\title{
A CIRCUNFERÊNCIA DA CINTURA ESTÁ ASSOCIADA A PRESENÇA DE INCAPACIDADE FÍSICA-FUNCIONAL EM IDOSOS?
}

Janaina Rocha Niehues - Programa de Pós-Graduação em Ciências da Reabilitação - PPGCR da Universidade Federal de Santa Catarina (UFSC) Campus Araranguá, Araranguá - SC, Brasil. Email: jananiehues@gmail.com;

Rafaela Aguiar Rosa - Graduanda do Curso de Fisioterapia na Universidade Federal de Santa Catarina (UFSC) Campus Araranguá, Araranguá - SC, Brasil. Email: rafaelaaguiarrosa14@gmail.com;

Núbia Carelli Pereira de Avelar - Docente do Curso de Fisioterapia do Departamento de Ciências da Saúde do Centro de Ciências, Tecnologias e Saúde do Campus Araranguá. Email: nubia.carelli@ufsc.br;

Ana Lúcia Danielewicz - Programa de Pós-Graduação em Ciências da Reabilitação - PPGCR da Universidade Federal de Santa Catarina (UFSC) Campus Araranguá, Araranguá - SC, Brasil. Docente do Curso de Fisioterapia do Departamento de Ciências da Saúde do Centro de Ciências, Tecnologias e Saúde do Campus Araranguá. Email: ana.lucia.d@ufsc.br

\section{RESUMO}

Introdução: $\mathrm{O}$ envelhecimento é associado à redução na massa muscular e no gasto energético basal que predispõe ao acúmulo de gordura predominantemente na região abdominal. Além disso, a redução do tecido muscular associa-se a incapacidade física-funcional. Torna-se necessário verificar a associação entre circunferência da cintura (CC) e incapacidade física-funcional para o estabelecimento de estratégias preventivas da obesidade. Objetivo: Verificar a associação entre CC e a incapacidade física-funcional em idosos. Métodos: Tratou-se de um estudo transversal, aleatório, de base domiciliar, no qual participaram 308 idosos ( $\geq 60$ anos), do município Balneário Arroio do Silva/SC. A incapacidade funcional nas atividades básicas de vida diária (ABVDs) foi avaliada pelo autorrelato de pouca/muita dificuldade ou impossibilidade para realizar ao menos uma tarefa (comer, vestir-se, tomar banho, usar o banheiro, andar no plano e deitar-se). A limitação na mobilidade foi avaliada pelo Timed Get Up and Go ( $\geq 10 \mathrm{~s}=$ limitação de mobilidade). Valores $>102 \mathrm{~cm}$ para homens e $>88$ $\mathrm{cm}$ para mulheres foram categorizados como altos valores de CC. Foi realizado teste de Qui-quadrado de Pearson. Resultados: Na amostra avaliada 57,8\% eram mulheres com média de idade de $69,86 \pm 7,06$ anos. As prevalências observadas foram 68,9\% (IC95\%: 63,5;73,9) de altos valores de CC, 48,2\% (IC95\%: 42,6;53,8) de incapacidade nas ABVDs e 33,2\% (IC95\%: 27,9;38,9) de limitação na mobilidade. A maioria dos idosos com altos CC (51,7\%) apresentaram incapacidade nas ABVDs $(p=0,047)$ e 37,3\% apresentaram limitação de mobilidade $(p=0,037)$. Conclusão: A CC foi associada a presença de incapacidade nas ABVDs e de limitação na mobilidade nos idosos amostrados.

Palavras-chave: obesidade abdominal; atividades cotidianas; limitação da mobilidade. 